expert clinical judgement, followed by validation against case note review $(\mathrm{n}=1058)$.

Results The algorithm developed had sensitivity $74 \%$ (95\% CI 69\% to $78 \%$ ) and estimated specificity was $94 \%$. Applied to all Scottish hospital activity data for $2005(\mathrm{n}=883 \mathrm{~K})$, the algorithm gave an estimate of annual incidence of severe sepsis (2.7\%) and case mortality (34\%). Analyses were undertaken of factors associated with severe sepsis and outcomes. For example, it was found that in those with severe sepsis, critical care admission was less common in females and those aged over 70 years.

Conclusion Internationally, this is the first rigorously-validated algorithm to detect severe sepsis, and performance is impressive given the complex nature of the condition. Application of the algorithm to provide reliable hospital-wide case rates will allow monitoring of incidence and outcomes, and better-informed planning of intensive care services.

\section{P1-62 THE VIRTUAL COMMITTEE: A PRACTICAL PROCESS FOR MAINTAINING HIGH OUALITY CONTENT OF ONLINE LEARNING RESOURCES FOR PUBLIC HEALTH PRACTICE IN CANADA}

doi:10.1136/jech.2011.142976c.55

E Wright, ${ }^{*}$ H Robinson, J Rossiter. Public Health Agency of Canada, Ottawa, Ontario, Canada

Introduction The Public Health Agency of Canada's Skills Online program, developed in partnership with government, academic and professional organizations, supports public health core competencies by enhancing the skills of practitioners. This internet-based continuing education program aims to provide relevant, timely and practical Canadian content for public health practitioners. It is delivered in English and French, with an international component. More than 6000 individual public health practitioners have taken at least one of the modules. Subject areas include basic and epidemiology, outbreak management, surveillance, and evidence based public health practice (http://www.phac-aspc.gc.ca/skills). A significant challenge for the program is how best to maintain high quality content that reflects the current realities of Canadian and international public health practice, using up-to-date educational approaches, all informed by a variety of public health practitioner experiences.

Methods The Public Health Agency of Canada has met this challenge by establishing a multidisciplinary committee, comprised of public health experts, continuing education experts, practitioners and program participants. This "virtual committee" uses a variety of communication options to permit monthly virtual meetings, supplemented by online sessions and demonstrations, and occasional face-to-ace meetings.

Results This approach has resulted in a dynamic process for the continued review and revision of the content of online learning modules, establishment of some innovative learning solutions, and ongoing evaluation of the program.

Conclusion A virtual committee efficiently and effectively provides for maintenance of content, essential for the Public Health Agency of Canada's continued delivery of high quality continuing education for public health practitioners.

\section{P1-63 ESTIMATING FRACTION CURED FROM CANCER: WHICH STATISTICAL PACKAGE TO USE?}

doi:10.1136/jech.2011.142976c.56

${ }^{1,2} \mathrm{X} O \mathrm{Yu},{ }^{*},{ }^{4} \mathrm{M}$ Clements, ${ }^{1} \mathrm{D}$ 0'Connell. ${ }^{1}$ Cancer Council New South Wales, Sydney, Australia; ${ }^{2}$ The University of Sydney, Sydney, Australia; ${ }^{3}$ Australian National University, Canberra, Australia; ${ }^{4}$ Karolinska Institutet, Stockholm, Sweden

Background Estimates of the fraction of patients cured from cancer provide important information to both patients and clinicians. But how reliable are the estimates?
Methods Three statistical approaches, based on similar assumptions that a fraction of patients will be cured from cancer, were used to estimate the fraction cured. The first approach was CANSURV software and the second was developed by De Angelis et al (1999), both using grouped survival data. The third was published by Lambert et al (2007), requiring individual patient records. All three approaches fit mixture cure models; and CANSURV and Lambert's implementation use maximum likelihood, while De Angelis' implementation is based on non-linear least squares. Cansurv is a standalone program whereas the other approaches were implemented using SAS and Stata respectively. SEER-9 data for rectal cancer were used to illustrate the methods.

Results As shown in the Abstract P1-63 table 1, estimates of the cure fraction were similar for the two approaches requiring grouped survival data while Lambert's method provided lower a estimate for patients with localised disease.

\section{Abstract P1-63 Table 1}

\begin{tabular}{llll}
\hline $\begin{array}{l}\text { Estimated } \\
\text { cure fraction }\end{array}$ & Cansurv & $\begin{array}{l}\text { De Angelis } \\
\mathbf{1 9 9 9}\end{array}$ & $\begin{array}{l}\text { Lambert } \\
\mathbf{2 0 0 7}\end{array}$ \\
\hline Localised & 0.720 & 0.738 & 0.667 \\
Regional & 0.404 & 0.406 & 0.388 \\
Distant & 0.049 & 0.033 & 0.034 \\
\hline
\end{tabular}

Discussion The three approaches provided similar estimates of the cure fraction for patients with regional and distant stage at diag nosis, however there are considerable differences in the estimates for patients with localised disease. Estimates of the cure fraction appear to depend on the choice of statistical model even when the underlying assumptions are very similar.

\section{P1-64 NOVEL GENETIC RISK VARIANTS FOR BREAST CANCER: FROM DISCOVERY TO DISEASE PREVENTION}

doi:10.1136/jech.2011.142976c.57

W Zheng. ${ }^{*}$ Vanderbilt University, School of Medicine, Nashville, Tennessee, USA

Breast cancer is the most common malignancy among women in many parts of the world. Genetic factors play an important role in the aetiology of this common malignancy. Recent genome-wide association studies (GWAS) have identified multiple genetic susceptibility loci for breast cancer. Most of the GWAS, however, were conducted among women of European ancestry. It is unclear whether genetic risk variants identified in Europeans may also be associated with breast cancer risk in Asians. Furthermore, genetic factors identified to date explain only a small fraction of heritability for breast cancer, and thus many genetic susceptibility loci remain to be discovered. In 2008 we established the Asia Breast Cancer Consortium to search for genetic risk variants for breast cancer. The consortium now consists of over 15000 breast cancer cases and an equal number of controls who were recruited from 12 studies conducted among Chinese, Korean, and Japanese women. Using a multi-stage study design, we have identified several novel genetic risk variants for breast cancer, including those located at chromosome $6 \mathrm{q} 25$ and $16 \mathrm{q} 12$. We also have systematically evaluated GWAS-reported genetic risk variants in Chinese and established a risk prediction model that incorporates information from both traditional clinical predictors and genetic risk markers. Results from the Asia Breast Cancer Consortium demonstrate the value of conducting genetic studies in non-European populations to identify novel genetic factors for breast cancer. 